\title{
Stable and Passive High-Power Dual Active Bridge Converters Interfacing MVDC Grids
}

F. D. Freijedo, E. Rodriguez-Diaz, and D. Dujic

This material is posted here with permission of the IEEE. Such permission of the IEEE does not in any way imply IEEE endorsement of any of EPFL's products or services. Internal or personal use of this material is permitted. However, permission to reprint / republish this material for advertising or promotional purposes or for creating new collective works for resale or redistribution must be obtained from the IEEE by writing to pubs-permissions@ieee. org. By choosing to view this document, you agree to all provisions of the copyright laws protecting it. 


\title{
Stable and Passive High-Power Dual Active Bridge Converters Interfacing MVDC Grids
}

\author{
Francisco D. Freijedo, Senior Member, IEEE, Enrique Rodriguez-Diaz, Student Member, IEEE, \\ and Drazen Dujic, Senior Member, IEEE
}

\begin{abstract}
Dual active bridge (DAB) is a topology that is receiving more and more attention as a potential solution to interface dc grids of different voltage levels. From a system level, the implications of DABs on the stability of complex power systems are addressed in this work. Dynamics modeling and stability assessment for a DAB implementation aimed to interface low-voltage energy resources with a medium-voltage dc (MVDC) collection and distribution grid are presented. The DAB admittance is analytically derived and assessed in order to describe its dynamics and anticipate its behavior when integrated in a complex MVDC grid. The model considers the low frequency range, mostly dominated by the controller action, and the high frequency range, described by a non-linear operation. The theoretical analysis is verified by hardware-in-the-loop emulation, with the controller running on a digital signal processor. The proposed implementation is proved to achieve passivity in the whole spectrum, which undoubtedly is a desired feature for a massive power electronics integration in the future MVDC grids.
\end{abstract}

Index Terms-Admittance measurement, dc-dc power conversion, power generation control, stability.

\section{INTRODUCTION}

Medium voltage dc (MVDC) collection and distribution grids are being considered for different applications such as renewable energy, big data centers, marine power systems and microgrids. Efficiency, size and cost are among the main reasons for the move from ac to dc [1]-[4].

As a quick overview to show the benefits of dc grids, Fig. 1 shows a benchmark case for comparison between a conventional ac system versus a prospective dc one. Fig. 1(a) depicts one of the most employed configurations for distributed generation, such as wind turbines [5]. A low voltage (LV) synchronous machine is connected to the grid system through a back to back converter; for multi-MW solution, paralleling of full-converters is also a prevalent solution [5]. Interface with distribution system is made by a bulky $50 / 60 \mathrm{~Hz}$ stepup transformer. Usually, multiple distributed generation (DG) resources are connected to a common medium voltage ac

Manuscript received October 26, 2017; revised January 24, 2018 and February 27, 2018; accepted March 11, 2018.

This work was supported in part by the Swiss Federal Office of Energy under the Granted Project "Medium-Voltage Direct-Current Energy Conversion Technologies and Systems (SI/501259)".

Francisco D. Freijedo (corresponding author) and Drazen Dujic are with the Power Electronics Laboratory, EPFL, CH1005, Lausanne, Switzerland. E-mails: francisco.freijedo@epfl.ch (Tel: +41216934789 and Fax: +412169326 00) and drazen.dujic@epfl.ch.

Enrique Rodriguez-Diaz is with the Department of Energy Technology, AAU, 9220 Aalborg East, Denmark. E-mail: erd@et.aau.dk.

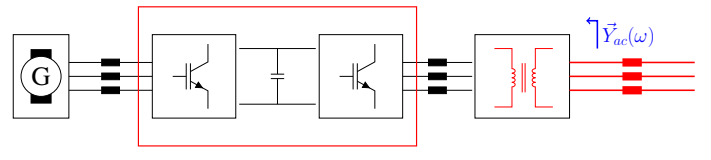

(a)

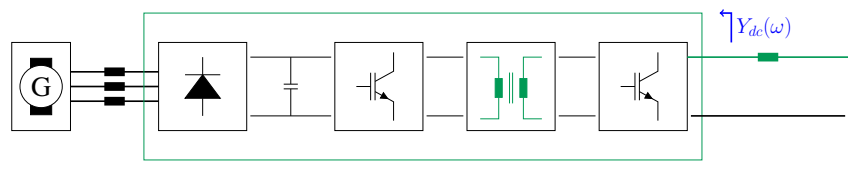

(b)

Fig. 1. A benchmark for ac vs dc comparison; LV electric machine and connection to a MV distribution line. a) Conventional solution based on LVAC power electronics and step-up transformer. b) Solution based on DAB which directly interfaces MVDC distribution.

(MVAC) distribution system, with cable impedances also playing a role in terms of losses and dynamics [6]. Using the same electric machine in the generation, an equivalent system to interface MVDC distribution is represented in Fig. 1(b). A controlled or uncontrolled rectifier can be employed to convert ac to dc at the LV side [7], [8]. Subsequently a dual active bridge (DAB) is considered in this work to deliver active power from low voltage dc (LVDC) to MVDC. The DAB is formed by two full bridges (FB) and a medium frequency transformer (MFT). MVDC distribution is represented by a single-line, regardless of an unipolar or bipolar distribution implementation. From inspection of Fig. 1, some MVDC benefits can be identified:

1) Enhanced efficiency: typical curves for full-converter based wind turbines show a maximum efficiencies around the nominal power of around 97\% [9]. MVDC technology is expected to improve this number: e.g., efficiencies near $99 \%$ have been reported for the DAB power conversion stage [10]-[12].

2) Size, weight and cost reductions in distribution cables: for a same power level, a dc cable based distribution increase by a 1.56 factor the power density of a conventional threewire ac system [10]. Intuitive explanations are i) from a peak voltage definition, dc voltage has a $\sqrt{2}$ times higher $r m s$ value than an ac one; ii) the absence of inductive/capacitive effects in dc distribution.

3) Size, weight and cost reductions in magnetic components: MVDC technology relies on MFT to step up from LV to MV. MFTs provide a much higher power density than line frequency transformers (LFTs) [13], [14]. The removal of bulky LFTs and its substitution by much more compact 
components is a key advantages of MVDC [2], [7].

Recent contributions show enabling technologies for MVDC grids: i) high power dc-dc converters, based on different device technologies (i.e., IGBTs, IGCTs, SiC MOSFETs), have been validated in controlled environments [10], [15]-[18]. ii) MFTs with a high power density are also reported [13], [14], [19]. iii) solutions at a power system level have been explored [1], [8], [20], [21]. iv) a bottleneck for MVDC technology is the lack of commercially available protection devices [22]-[24]; efforts to develop cost-effective protection solutions have been provided, recently [24], [25].

Another key concern of MVDC technology, explicitly addressed in this work, is the system level stability. An impedance stability approach is used as natural framework to evaluate addition of DG resources: stability properties of individual DG resources can be studied by their corresponding $\vec{Y}_{a c}(\omega)$ frequency response [26]-[34]. More specifically, inputadmittance passivity compliance provides a sufficient condition for stability and is also considered a good indicator of the robustness of the system [15], [26], [27], [32]-[34]. In a similar fashion as in ac system analysis, dynamic properties can be evaluated by $Y_{d c}(\omega)$ admittance shaping [35], [36]. Therefore, in this work, a design for passivity methodology is provided and passivity compliance is selected as a main figure of merit to assess stability and robustness.

The paper structure is as follows. Section II shows a description of the DAB based solution and provides controller design guidelines. Section III develops admittance models in low and high frequency regions of the spectrum. Section IV shows experimental results obtained from hardware-in-theloop (HIL) emulation. Finally the main results and properties of the analyzed system are discussed and summarized.

\section{System DESCRIPTION}

Fig. 2 shows a simplification suitable for the MVDC collection and distribution of the DC wind farm concept analyzed in [2], [7], [8]. The lumped admittance model that groups all the wind turbine generators is defined as $Y_{G}^{\text {total }}(\omega)$, with $Y_{\text {WT_1 }}(\omega), Y_{\text {WT_2 }}(\omega)$ (and so on) defining individual wind turbine systems. Mathematically,

$$
Y_{G}^{\mathrm{total}}(\omega)=\sum_{k=1,2, \ldots}^{N} Y_{\mathrm{WT}_{-} k}(\omega) .
$$

From (1), $Y_{G}^{\text {total }}(\omega)$ is defined passive if all its elements are defined passive. In this work, the wind turbines models are based on Fig. 3(a), with $Y_{2}(\omega)$ defining an individual admittance in the MVDC side [e.g., $Y_{\text {WT_1 }}(\omega)$ in Fig. 2].

Fig. 3 represents the power circuit of the DAB based generation system for the analysis and its corresponding control diagrams. The hardware parameters are shown in Table I. In a practical implementation, the full bridge in the MVDC side includes series connected IGBT/IGCT devices to reach the desired voltage level [10]. The leakage inductance has been calculated from the phase-angle versus active power relation [cf. (2)] and considering a maximum angle for 1.5 times the nominal power. The LVDC source is obtained by a rectifier connected at the stator terminals of the DG electrical machine.

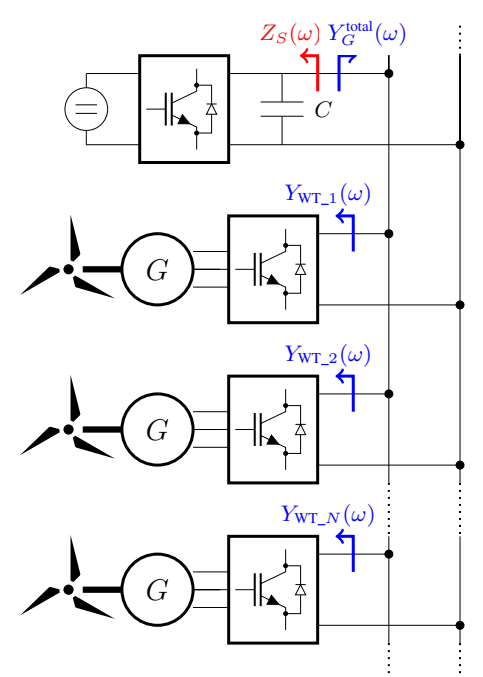

Fig. 2. Simplified MVDC distribution system of a DC wind farm.

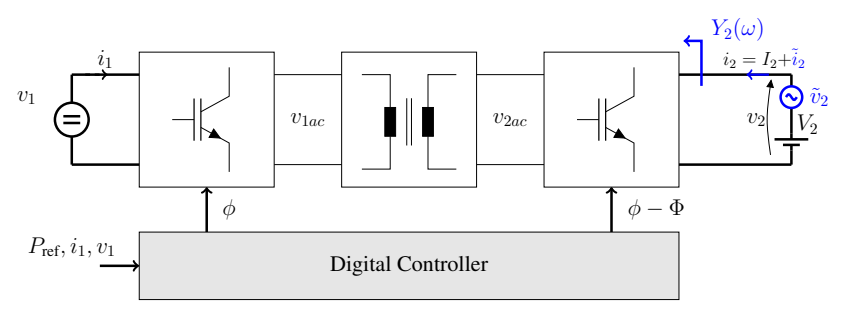

(a)

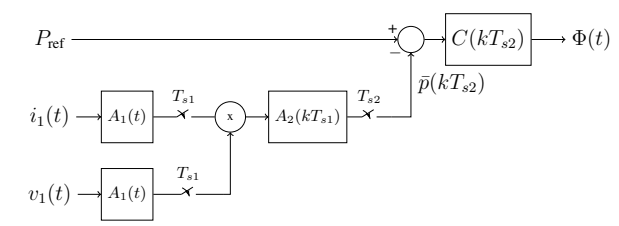

(b)

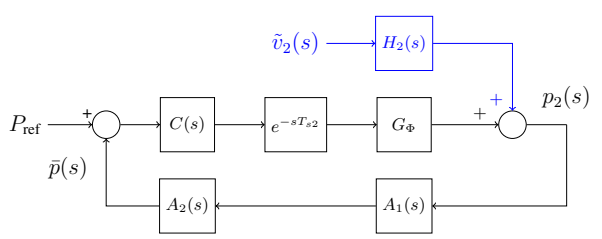

(c)

Fig. 3. Proposed system. a) DAB circuit. b) Proposed controller implementation: $A_{1}(t), A_{2}\left(k T_{s 1}\right), C\left(k T_{s 2}\right)$ represent the anti-aliasing filters, a low pass filter to smooth power measurement and the main controller, respectively. c) Low frequency linearized model of the closed-loop; in blue the $\tilde{v}_{2}(s)$ perturbation path.

Therefore, the average value and ripple may depend on the speed and active power from the wind turbine generator [8], which is explicitly considered in the HIL validation (cf. section IV). The DAB second stage delivers active power to the MVDC distribution grid. For $Y_{2}(\omega)$ modeling, the MVDC grid is assumed to be an ideal dc source in series with a smallsignal sinusoidal perturbation. $Y_{2}(\omega)$ compliance with the passivity property is employed to assess the stability properties of the system integration into a MVDC grid (cf. section III-C for the specific details and discussion). 
TABLE I

CiRCUIT PARAMETERS

\begin{tabular}{cc}
\hline Rated Power of DG resource & $P_{\text {rated }}=2 \mathrm{MW}$ \\
\hline LVDC nominal voltage & $V_{1}=1100 \mathrm{~V}$ \\
\hline MVDC nominal voltage & $V_{2}=20 \mathrm{kV}$ \\
\hline MFT (nominal) transformation ratio & $n=V_{1} / V_{2}=0.055$ \\
\hline PWM Carrier period (frequency) & $T_{c}=250 \mu \mathrm{s}\left(f_{c}=4 \mathrm{kHz}\right)$ \\
\hline MFT leakage inductor & $L_{\sigma}=12.6 \mathrm{uH}$ \\
\hline MFT leakage resistor & $R_{\sigma}=31 \mathrm{~m} \Omega$ \\
\hline
\end{tabular}

\section{A. Controller Design Guidelines}

In most of distributed generation systems, such as wind turbines, the electric drive train acts as an physical actuator of a higher level mechanical controller. Power control operation is a suitable interface between mechanical and electric systems. In wind turbine applications, the controller design requirements are usually expressed in terms of power command step tracking: say i) a first order response is required (i.e., overshoot is not allowed to avoid mechanical stress); ii) a maximum allowable time constant is specified (i.e., the electric drive train should be faster than other mechanical actuators). A conservative value for the maximum time constant allowed (for a power command step) $\tau_{P}$ is $100 \mathrm{~ms}$ [37]. An even more restrictive value is selected in this paper as shown in Table II.

Power control by phase-shift angle modulation and control for DAB is considered [38], [39]. Assuming $v_{1}(t)=\bar{v}_{1}(t)=$ $V_{1}$ and $v_{2}(t)=\bar{v}_{2}(t)=V_{2}$, for a carrier period, the average transferred power equation is given by

$$
\begin{aligned}
<p(\Phi, t)>_{T_{c}} \equiv P(\Phi)= & \frac{T_{c} V_{1} n V_{2}(\pi-\Phi) \Phi}{2 \pi^{2} L_{\sigma}} \\
& \forall 0 \leq \Phi \leq \pi / 2
\end{aligned}
$$

with $T_{c}$ being the carrier frequency and $\Phi$ the relative angle between the voltages at the MFT terminals [c.f., $v_{1 a c}$ and $v_{2 a c}$ in Fig. 3(a)]. This is a non-linear expression that sets a dc-bias operating point.

Closed loop power regulation of a DAB can be achieved by considering $\Phi(t)$ as an actuation variable [as shown in Fig. 3(a), $\phi(t)=\int \omega_{s} t$ is the same for both converters] [38], [39]. By small signal perturbation of (2) around a given operation point, the following linear relation is obtained

$$
\Delta P(t)=\frac{T_{c} V_{1} n V_{2}[\pi-\Phi]}{2 \pi^{2} L_{\sigma}} \Delta \Phi(t) \quad \forall 0 \leq \Phi \leq \pi / 2
$$

with $\Delta \Phi(t)$ and $\Delta P(t)$ being the small-signal perturbations of phase-shift angle and power transferred, respectively. By applying the Laplace transformation of (3), at each specific operation point, the plant of the system is defined as

$$
\Delta P(s)=G_{\Phi}\left(P_{\text {in }}\right) \Delta \Phi(s)
$$

with

$$
\underbrace{\frac{T_{c} V_{1} n V_{2}}{4 \pi L_{\sigma}}}_{G_{\Phi_{\text {min }}}} \leq G_{\Phi}\left(P_{\text {in }}\right) \leq \underbrace{\frac{T_{c} V_{1} n V_{2}}{2 \pi L_{\sigma}}}_{G_{\Phi_{\max }}} .
$$

TABLE II

Controller Parameters

\begin{tabular}{cc}
\hline Required $P_{\text {ref }}$ tracking & $1^{s t}$ order, $\max \left(\tau_{P}\right)=50 \mathrm{~ms}$ \\
\hline Acquisition sampling period & $T_{s 1}=125 \mu \mathrm{s}$ \\
\hline Controller sampling period & $T_{s 2}=1.25 \mathrm{~ms}\left(0.1 T_{s 1}\right)$ \\
\hline Anti-aliasing LPF & $2^{\text {nd }}$ order, $\zeta=1, \omega_{n}=5000 \mathrm{rad} / \mathrm{s}$ \\
\hline Power measurement LPF & $1^{s t}$ order, $\tau_{M}=100 \mathrm{~ms}$ \\
\hline Controller gains & $k_{p}=1.645 e-06 \mathrm{rad} / \mathrm{W}$, \\
& $k_{i}=1.645 e-05 \mathrm{rad} /(\mathrm{s} \cdot \mathrm{W})$, \\
{$[$ From $(7)$ with $\alpha=2 \pi 5 \mathrm{rad} / \mathrm{s}]$} \\
\hline
\end{tabular}

Clearly, the controller gains should be selected to comply with the requirements in the range of gains defined by (5).

Since the accuracy of (3) is, by definition, only assured in the range of frequencies defined by $\omega<<1 / T_{c}$, a bandlimitation of the controller should be considered in order to assure a linear behavior. This is done by down-sampling the control rate to $T_{s 2}>>T_{c}$. Even though extra time delay is introduced with down-sampling, in practice this is not a limitation, because the dynamic requirements from a mechanical systems are of several orders of magnitude below DAB PWM frequencies (i.e., $\tau_{P}>>T_{s 2}>>T_{c}$ in practice). It should be noticed that, as a consequence of down-sampling, two different sampling rates are employed. A fast acquisition period $T_{s 1}$ of twice $T_{c}$ (double update) in combination with analog anti-aliasing filter $A_{1}(s)$ is needed to avoid aliasing [by nature $i_{1}(t)$ is pulsating]. $A_{2}\left(k T_{s 1}\right)$ is a reduced bandwidth low pass filter of the first order, which smooths the power feedback signal. In practice, $A_{2}\left(k T_{s 1}\right)$ is implemented with much slower dynamics than the one of $A_{1}(t)$. Therefore, the $A_{1}(t)$ dynamics can be neglected in the calculations of a proportional-integral (PI) controller gains, which are performed as follows. Firstly, $A_{2}\left(k T_{s 1}\right)$ is approximated to a continuous domain filter as

$$
A_{2}(s)=\frac{1}{\tau_{a 2} s+1}
$$

Subsequently, by applying internal model laws [40] with the smallest gain, the controller gains are expressed as follows.

$$
k_{p}=\frac{\alpha \tau_{a 2}}{G_{\Phi_{\text {min }}}} \text { and } k_{i}=\frac{\alpha}{G_{\Phi_{\text {min }}}}
$$

with $\alpha$ being the intended bandwidth, which is constrained by $1 / \alpha<\max \left(\tau_{P}\right)$ and $1 / \alpha>>T_{s 2}$.

Fig. 4 depicts frequency diagrams from the controller design. It is clear that from the range of operation between no load and $P_{\text {rated }}$, the operation point is kept almost constant, which is a good indicator for a linear response of the system. The closed loop bandwidth is above $\alpha$, which assures to comply with the main dynamic constraint.

The proposed DAB power control is based on a single-loop with an imposed bandwidth limitation. The objective of this strategy is to avoid closed-loop operation in the high frequency range, where the in-loop delays would tend to make passivity compliance unfeasible (references [32], [34] analyze in detail how system delays effects compromise input-admittance passivity compliance, in ac grid-connected converters). 


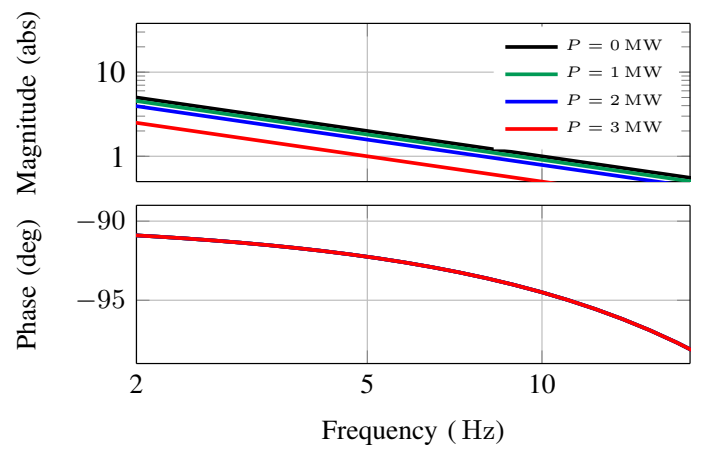

(a)

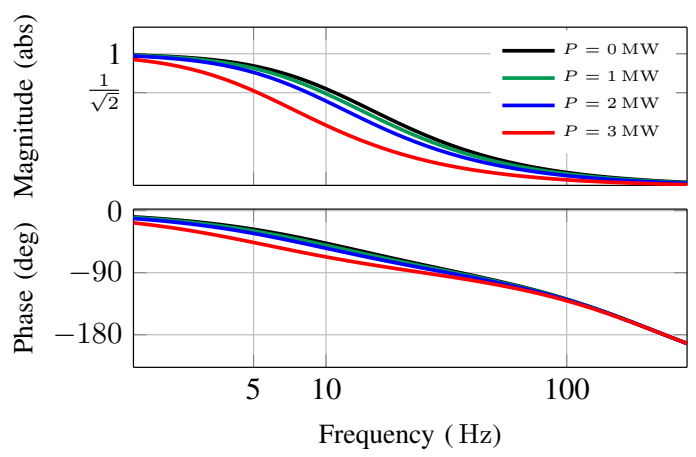

(b)

Fig. 4. Controller design diagrams for the linearized model in Fig. 3(c) a) Open loop gain. b) Closed loop $P_{\text {ref }}$ tracking.

\section{Admittance Calculation}

The admittance $Y_{2}(\omega)$ [i.e., the admittance as seen from the DAB connection point to the MVDC grid, as depicted in Figs. 1(b) and 3(a)] is derived considering a small signal perturbation in $v_{2}(t)$, and how this is reflected in power $p_{2}(t)$ and current $i_{2}(t)$. The power equation in the MVDC side is

$$
p_{2}(t)=v_{2}(t) i_{2}(t)
$$

For a given operation point,

$$
\begin{gathered}
v_{2}(t)=V_{2}+\tilde{v}_{2}(t) \\
i_{2}(t)=I_{2}+\tilde{i}_{2}(t) \\
p_{2}(t)=P_{2}+\tilde{p}_{2}(t)
\end{gathered}
$$

with $V_{2}, I_{2}$ and $P_{2} \equiv V_{2} I_{2}$ representing a dc-bias point, and $\tilde{v}_{2}(t), \tilde{i}_{2}(t)$ and $\tilde{p}_{2}(t)$ representing small-signal variables. By combining (8) and (9), the small-signal power equation is

$$
\tilde{p}_{2}(t)=I_{2} \tilde{v}_{2}(t)+V_{2} \tilde{i}_{2}(t) .
$$

Since (10) is linear, it can be expressed in the frequency domain

$$
\tilde{p}_{2}(s)=I_{2} \tilde{v}_{2}(s)+V_{2} \tilde{i}_{2}(s) .
$$

The admittance transfer function is available from (11),

$$
Y_{2}(s)=\frac{\tilde{i}_{2}(s)}{\tilde{v}_{2}(s)}=\frac{\tilde{p}_{2}(s) / \tilde{v}_{2}(s)-I_{2}}{V_{2}} .
$$

Therefore, the key step to obtain $Y_{2}(s)$ is to find the transfer function between $\tilde{p}_{2}(s)$ and $\tilde{v}_{2}(s)$. Analytical methodologies depend on the range of frequency of interest, as shown next.

\section{A. Open Loop Disturbance Model}

For $H_{2}(s)$ identification, the control action in Fig. 3(c) is disabled. The convolution theorem for Fourier transforms (cf. [41]) is the basis to calculate $H_{2}(\omega)$ : e.g., by applying the Convolution Theorem to (8),

$$
p_{2}(\omega)=v_{2}(\omega) * i_{2}(\omega) .
$$

The open loop disturbance frequency response, defined as $H_{2}(\omega)=\tilde{p}_{2}(\omega) / \tilde{v}_{2}(\omega)$, is obtained by perturbation analysis. A sinusoidal input perturbation, which is over-imposed to the dc bias voltage $V_{2}$, is defined in the complex time domain as

$$
\tilde{v}_{2}(t)=\frac{\hat{v}_{2}}{2}\left[e^{j\left(\omega_{p} t+\theta_{p}\right)}+e^{-j\left(\omega_{p} t+\theta_{p}\right)}\right]
$$

with $\hat{v}_{2}$ being amplitude, $\omega_{p}$ the perturbation frequency and $\theta_{p}$ an arbitrary phase-offset. By inspection of (14), $\tilde{v}_{2}(\omega)$ is a discrete Fourier series with two coefficients of amplitude $\hat{v}_{2} / 2$ and phase-angles $\pm \theta_{p}$, which are placed at $\pm \omega_{p}$ [41]. Fig. 5(a) represents $v_{2}(\omega)$ in the complex frequency domain, with the dc-bias component in black and the perturbation components in red [Fig. 5 is included to graphically support the different stages of $H_{2}(\omega)$ calculation].

A key step is to calculate the mapping of dc-side harmonics in the ac-side, for which an ideal modulator is considered for PWM modeling [41], which is described by

$$
\tilde{v}_{2 a c}(t)=\tilde{v}_{2}(t) \cdot \cos \left(\omega_{c} t\right)=\tilde{v}_{2}(t) \cdot \frac{e^{-j \omega_{c} t}+e^{j \omega_{c} t}}{2} .
$$

Equation (15) is indeed a simplification in the sense that it only calculates the dominant components that define the admittance, but neglects non-linear generation of other side-band harmonics (more accurate models based on PWM models are analyzed in [42], [43]). Fig. 5(b) shows the Fourier series coefficients from (15). The gain of the fundamental components (in black) has been corrected by a $4 / \pi$ term to consider the fact that $v_{2 a c}(t)$ is a square waveform of amplitude $V_{2}$ and frequency $\omega_{c}$; therefore, its fundamental component, employed in the Fourier series analysis, has an amplitude of $4 / \pi V_{2}$.

Subsequently, the ac current terms are calculated. The perturbation terms are given by

$$
\tilde{i}_{2 a c}(\omega)=\frac{\tilde{v}_{2 a c}(\omega)}{L_{\sigma}^{\prime} j \omega}
$$

with $L_{\sigma}^{\prime}=L_{\sigma} / n^{2}$ being the leakage inductance referred to the MV terminal ( $R_{\sigma}$ is neglected at this step). The $i_{2 a c}\left( \pm \omega_{c}\right)$ symmetrical coefficients are calculated in order to equal the power in MVAC and MVDC sides, i.e., from (13) and neglecting the small signal perturbation (in this step),

$$
v_{a c 2}\left(\omega_{c}\right) i_{a c 2}\left(-\omega_{c}\right)+v_{a c 2}\left(-\omega_{c}\right) i_{a c 2}\left(\omega_{c}\right)=V_{2} I_{2}
$$

The Fourier series coefficients of $i_{2 a c}(\omega)$ are depicted in Fig. 5(c). Fig. 5(d) shows the main ${ }^{1}$ Fourier coefficients of $p_{2 a c}(\omega)$, which are obtained from (13) and the Fourier coefficients of $v_{2 a c}(\omega)$ and $i_{2 a c}(\omega)$, depicted in Fig. 5(b) and

\footnotetext{
${ }^{1}$ Coefficients of $p_{2 a c}(\omega)$ of a frequency larger than $\omega_{c}$ are discarded.
} 


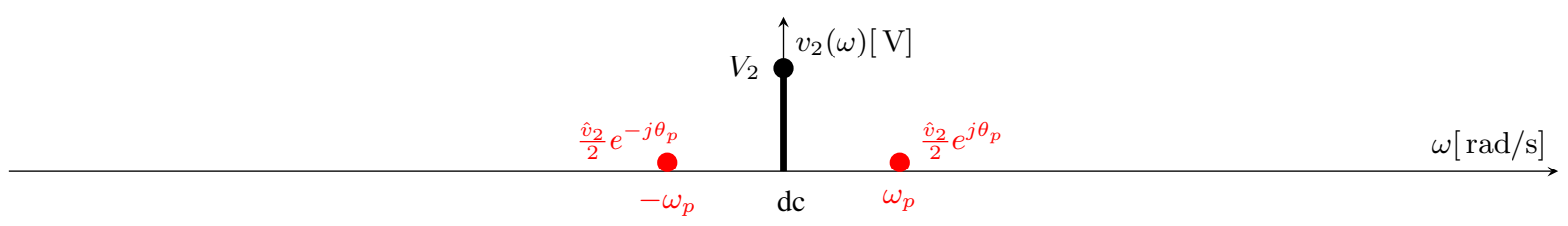

(a)

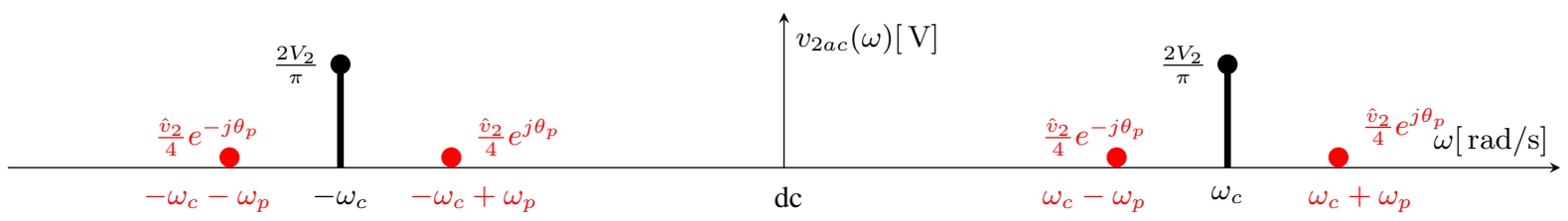

(b)

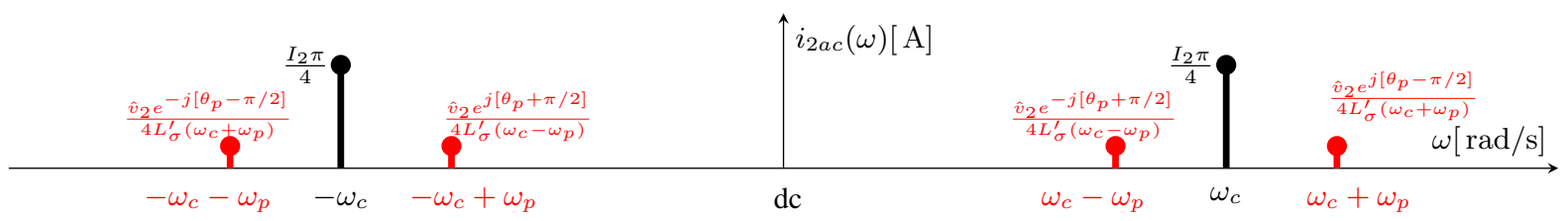

(c)

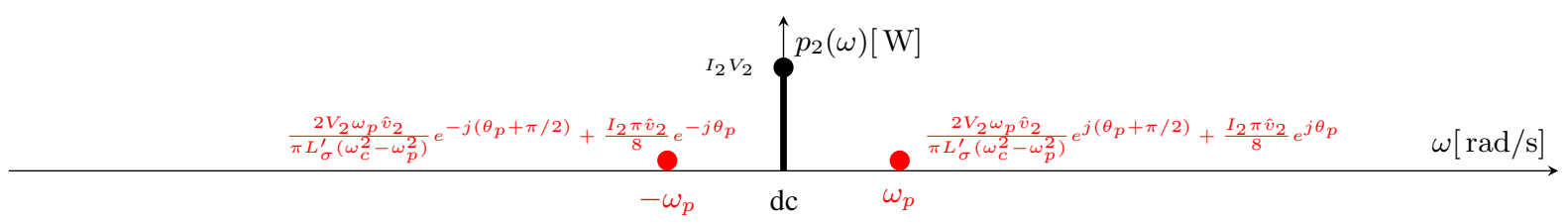

(d)

Fig. 5. Fourier Series coefficients of the signals involved in the high frequency model. Black bars correspond to dc-bias components. Red bars correspond to small-signal perturbation signals.

Fig. 5(c), respectively. It can be observed that, after convolution, $\tilde{p}_{2}\left(\omega_{p}\right)$ is linearly dependent on $\tilde{v}_{2}\left(\omega_{p}\right)$, and, therefore

$$
H_{2}\left(\omega_{p}\right) \equiv \frac{\tilde{p}_{2}\left(\omega_{p}\right)}{\tilde{v}_{2}\left(\omega_{p}\right)}=\frac{\pi I_{2}}{4}+j \frac{2 V_{2} \omega_{p}}{\pi L_{\sigma}^{\prime}\left(\omega_{c}^{2}-\omega_{p}^{2}\right)} .
$$

By using the Laplace variable $s=j \omega_{p}$, the open loop frequency model for the disturbance is given by

$$
H_{2}(s)=\frac{\pi I_{2}}{4}+\frac{2 V_{2} s}{\pi L_{\sigma}^{\prime}\left(s^{2}+\omega_{c}^{2}\right)} .
$$

\section{B. Closed Loop Model}

Taking into account the controller action, $\tilde{p}(s) / \tilde{v}_{2}(s)$ is obtained from the closed loop dynamics represented in Fig. 3(c). It can be appreciated that $\tilde{v}_{2}(s)$ is a disturbance of the power loop and, therefore, $\tilde{p}(s) / \tilde{v}_{2}(s)$ is the disturbance to signal transfer function, which can be obtained analytically as

$$
\begin{aligned}
\frac{\tilde{p}_{2}(s)}{\tilde{v}_{2}(s)} & =\frac{H_{2}(s)}{1+C(s) e^{-s T_{s 2} G_{\Phi}\left(P_{\text {in }}\right) A_{2}(s)}} \\
& =\frac{s H_{2}(s)}{s+\alpha e^{-s T_{s 2} \frac{G_{\Phi}\left(P_{\text {in }}\right)}{G_{\Phi_{\text {min }}}}} .}
\end{aligned}
$$

Subsequently, from (12)

$$
Y_{2}(s)=\frac{s\left[H_{2}(s)-I_{2}\right]-I_{2} \alpha e^{-s T_{s 2}} \frac{G_{\Phi}\left(P_{\text {in }}\right)}{G_{\Phi_{m i n}}}}{V_{2}\left[s+\alpha e^{-s T_{s 2}} \frac{G_{\Phi}\left(P_{\text {in }}\right)}{G_{\Phi_{\text {min }}}}\right]} .
$$

$Y_{2}(s)$ has a dc value defined by the operation point: $Y_{2}(0)=-I_{2} / V_{2}$ in steady-state. At high frequencies, disturbance and delay effects also shape the frequency response.

\section{Passivity Compliance}

$Y_{2}(\omega)$ compliance with the passivity criterion is established as stability and robustness figure of merit. This criterion has been previously adopted for certification of electric traction systems and can be also employed in the design of robust controllers for grid-connected converters [15], [26], [27], [32], [33]. The main feature of design for passivity is the fact that it provides a sufficient condition for stability, despite a big uncertainty in the grid model (environment). Mathematically, the passivity compliance is formulated as $-90 \operatorname{deg} \leq \angle Y_{2}(\omega) \leq 90 \mathrm{deg} \forall \omega$ or, equivalently, as $\operatorname{Real}\left\{Y_{2}(\omega)\right\}>0 \forall \omega$.

From (21), a maximum theoretical bandwidth to fulfill passivity criterion at nominal power conditions can be approximated as follows. Firstly, $\frac{G_{\Phi}\left(P_{\text {in }}\right)}{G_{\Phi_{\min }}} \approx \frac{G_{\Phi_{\max }}}{G_{\Phi_{\min }}}=2$ is a reasonable assumption [cf., Fig. 4(a)]. Also, $H_{2}(s) \approx \frac{\pi I_{2}}{4}$ is a very accurate assumption for the low frequency range. Equation (21) can be then simplified to

$$
Y_{2}(s) \approx \frac{-I_{2}}{V_{2}} \frac{s\left[\frac{4-\pi}{4}\right]+2 \alpha e^{-s T_{s 2}}}{s+2 \alpha e^{-s T_{s 2}}} .
$$




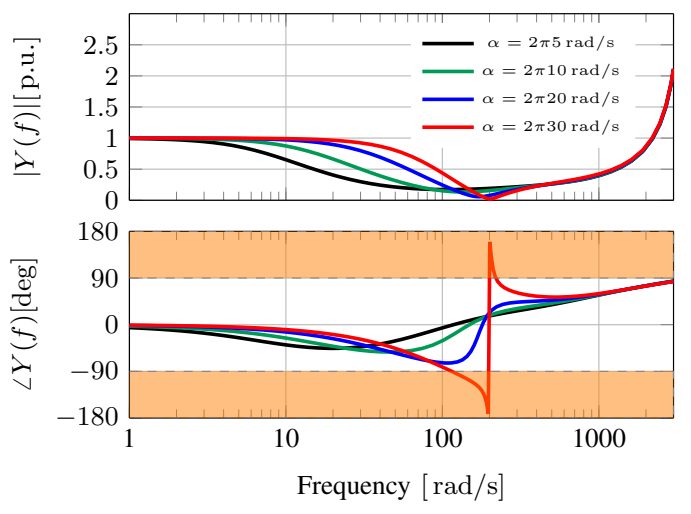

(a)

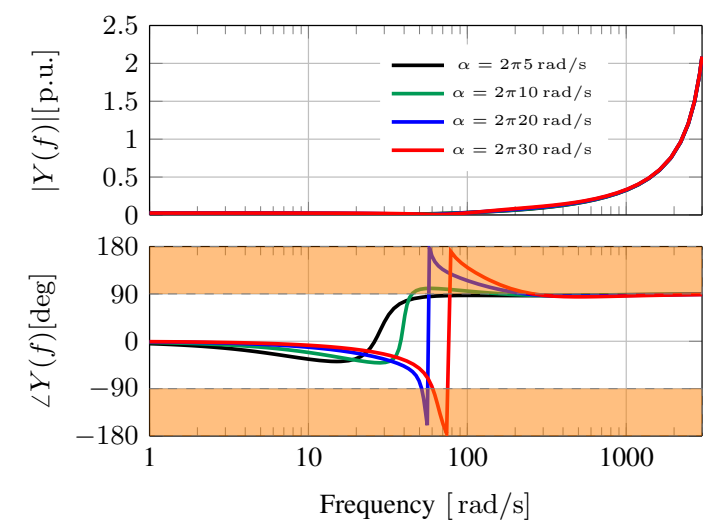

(b)

Fig. 6. Theoretical bandwidth limit for passivity compliance. a) Nominal power injection (2MW). b) $2.5 \%$ of nominal power injection.

An insightful approximation for the maximum achievable bandwidth (i.e., maximum $\alpha$ value that assures passivity compliance) can be identified by inspection of (22) at $\omega=\frac{\pi}{2 T_{s 2}}$, which is given by

$$
Y_{2}\left(j \omega=\frac{j \pi}{2 T_{s 2}}\right)=\frac{-I_{2}}{V_{2}} \frac{\frac{\pi}{2 T_{s 2}}\left[\frac{4-\pi}{4}\right]-2 \alpha}{\frac{\pi}{2 T_{s 2}}-2 \alpha} .
$$

Since $Y_{2}\left(j \omega=\frac{j \pi}{2 T_{s 2}}\right)=\operatorname{Real}\left\{Y_{2}\left(j \omega=\frac{j \pi}{2 T_{s 2}}\right)\right\}$, a limit condition for passivity is expected from a numerator sign change, which defines a maximum bandwidth as ${ }^{2}$

$$
\alpha_{\max } \equiv \frac{(4-\pi) \pi}{16 T_{s 2}} .
$$

Using the data of Table II in (24), $\alpha_{\max }=134.83 \mathrm{rad} / \mathrm{s}$ $(21.46 \mathrm{~Hz})$. Fig. 6(a) shows $Y_{2}(\omega)$ for different $\alpha$ values and proves the accuracy of (24) approximation. It can be also appreciated that, the passivity compliance can be achieved with a electrical response much faster than the one considered in section II-A. It can be also observed that $\alpha_{\max }$ sets a reasonable limit between a low frequency model (i.e., closed loop regulation is effective) and a high frequency one (i.e., open loop operation).

However, the accuracy of (24) would be compromised if a very low power set-point is employed, since the second

\footnotetext{
${ }^{2}$ The accuracy of this approximation is evaluated in the appendix.
}

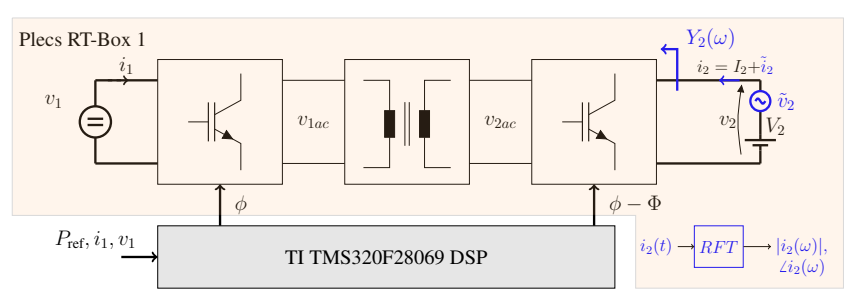

(a)

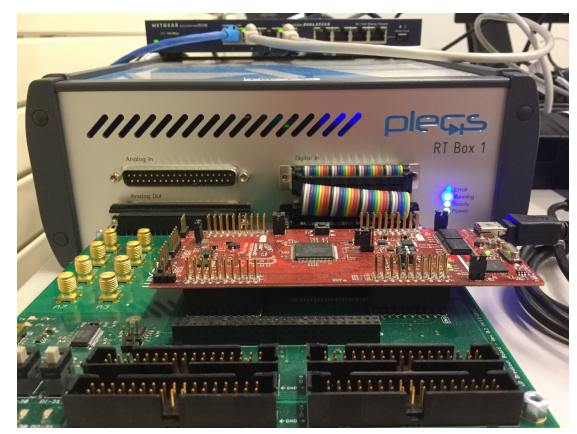

(b)

Fig. 7. Real time HIL experimental setup. (a) Model and controller implementation diagram. (b) Physical Devices.

order term in (19) becomes dominant when $I_{2} \approx 0$. A more restrictive theoretical bandwidth would be necessary to comply with the passivity criterion for very reduced power set-points. Referring to a typical wind turbine application, a minimum set-point is around $5 \%$ of the nominal power [44]. Fig. 6(b) predicts passivity compliance for an even smaller minimum power set point, such as $2.5 \%$ of the nominal power, using $\alpha_{\max }=2 \pi 5 \mathrm{rad} / \mathrm{s}$, which proves the suitability of the design obtained in section II-A).

\section{Real-Time HIL Results}

Fig. 7 shows the HIL based verification test-bed. The power hardware circuit, including switched model converters, is implemented in the RT-Box by Plexim, running at a $2.5 \mu \mathrm{s}$ cycle time. The controller has been implemented in a TI TMS320F28069 digital signal processor, running at $T_{s 1}=125 \mu \mathrm{s}$. The phase-shift angle $\Phi(t)$ of the MV side converter is implemented by the TBPHS register. TBPHS word length is 16 bits, but it range is limited to $[0, \pi / 2]$, so in practice the phase-angle shift has 14 bits of resolution (i.e., the minimum angle step change is $\Delta \Phi_{\min }=\frac{\pi}{2} \cdot \frac{1}{2^{-14}} \mathrm{rad}$ ).

Fig. 8 shows steady-state results at the rated power point. PWM1 and PWM2 firing signals correspond to the LV and MV half bridge, respectively [cf., $\phi$ and $\phi-\Phi$ in Fig. 3(a)]. The voltage drop in the leakage inductor and the ac current in LV side are also depicted.

Fig. 9 shows the time response for a positive and negative $P_{\text {ref }}$ steps, respectively. The proposed system achieves the $P_{\text {ref }}$ tracking requirements, despite the response is a bit slower than predicted. As expected from Fig. 4, a little dependence on the operation point is observed.

For each operation point, $Y_{2}(\omega)$ is calculated in real time. A voltage perturbation with known frequency and phase-angle offset $\hat{v}_{2}$ is added to the ideal MVDC source $V_{2}$. The amplitude 

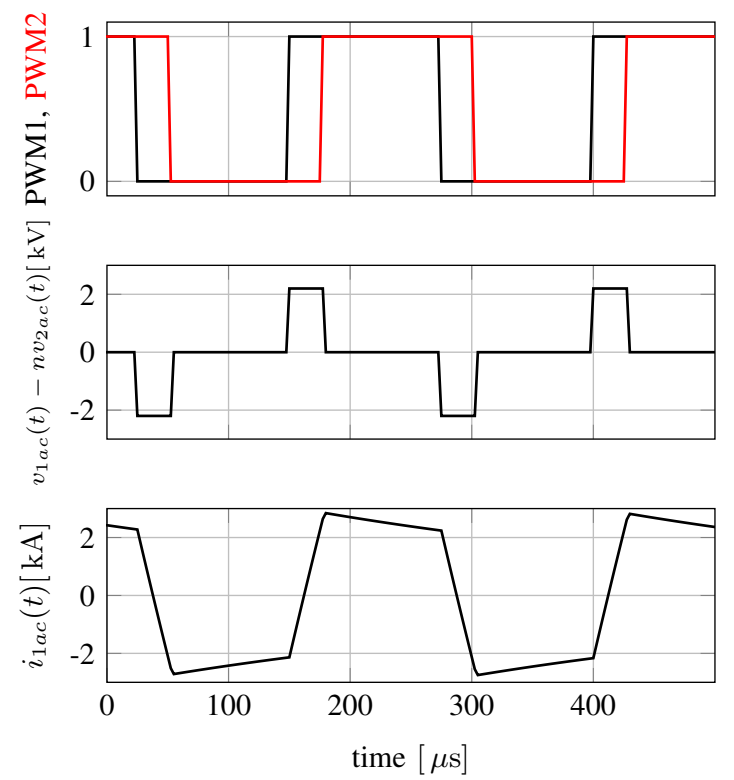

Fig. 8. Steady-state waveforms working at rated power ( $\left.P_{\text {ref }}=2 \mathrm{MW}, \Phi=39 \mathrm{deg}\right)$. With reference to Fig. 7(a), the phaseangles of PWM1 and PWM2 are $\phi(t)$ and $\phi(t)-\Phi(t)$, respectively.

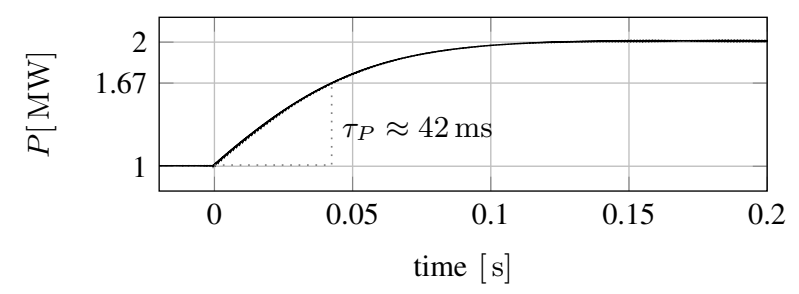

(a)

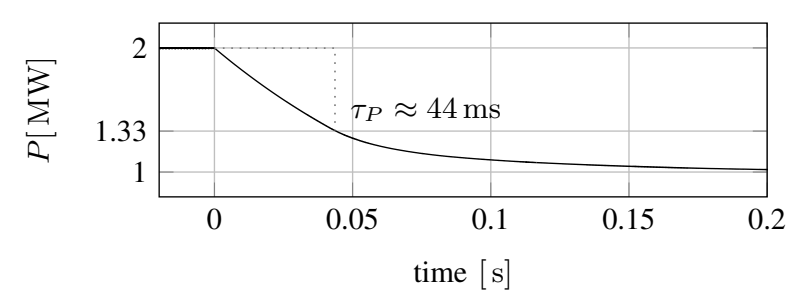

(b)

Fig. 9. Step response and time constant estimation. a) Response to a positive command step (from half to rated power). b) Response to a negative command step (from rated to half power).

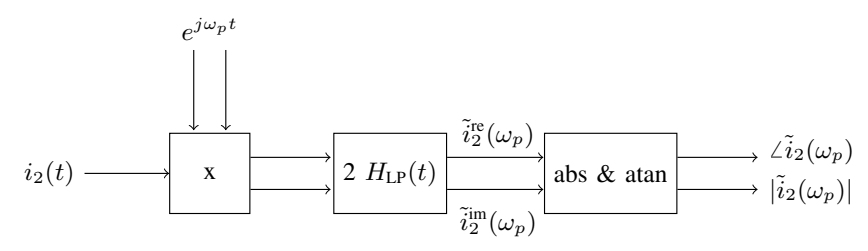

Fig. 10. Phase-angle and magnitude calculation for $\tilde{i}_{2}(\omega)$ based on a recursive calculation of Fourier coefficients [45].

of the small-signal perturbation $\hat{v}_{2}$ is 0.05 p.u.. Subsequently, amplitude and phase-angle of $\tilde{i}_{2}(\omega)$ are measured in real-

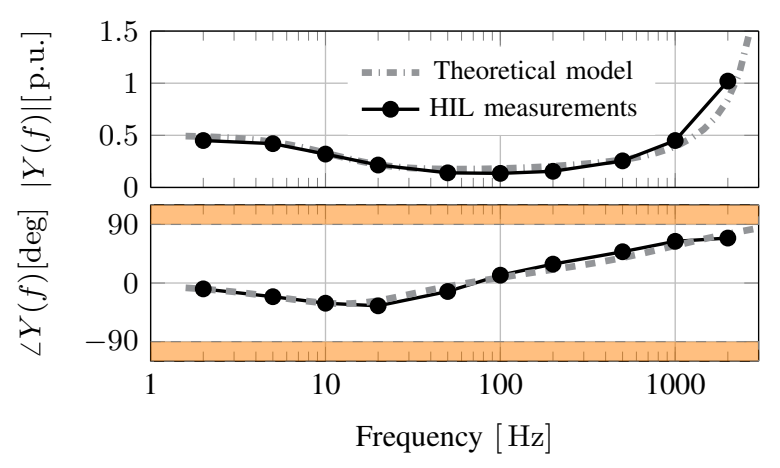

(a)

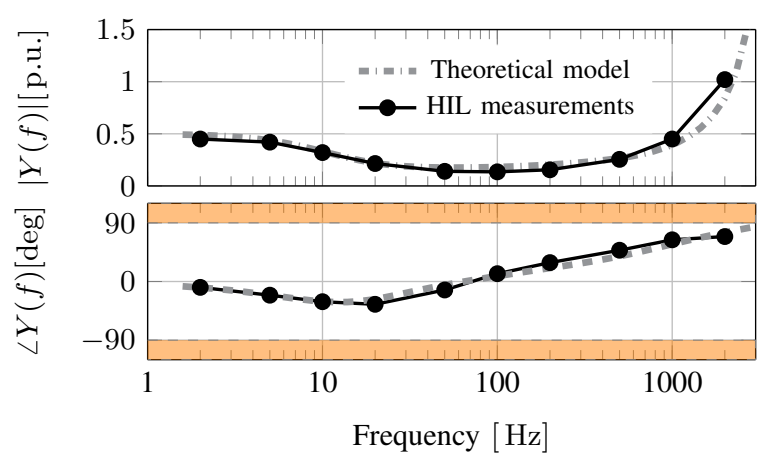

(b)

Fig. 11. Experimental results for $Y_{2}(\omega)$. The theoretical curves are obtained from (21). (a) operation at $2 \mathrm{MW}$. (b) operation at $1 \mathrm{MW}$.

time by recursive Fourier transform (RFT) [45], which is depicted in Fig. 10. Since an accurate calculation of low order harmonics is required, an infinite impulse low pass filter $H_{\mathrm{LP}}(s)$ with very reduced cut-off frequency $(<1 \mathrm{~Hz})$ is employed instead of moving average filters. The admittance at such frequency is given by

$$
Y_{2}(\omega)=\frac{\left|\tilde{i}_{2}(\omega)\right|}{\left|\tilde{v}_{2}(\omega)\right|}\left[\angle \tilde{i}_{2}(\omega)-\angle \tilde{v}_{2}(\omega)\right] .
$$

Key results are depicted in Fig. 11: $Y_{2}(\omega)$ is represented for full and half load, respectively. The real-time points closely match the predictions given by (21), which comprises both low and high frequency ranges. Passivity compliance is achieved in the whole spectrum, which, from system integration point of view, is undoubtedly a beneficial feature: i) inside a complex MVDC distribution system with multiple sources of background harmonics, the DAB would not amplify those ones and create further deterioration of the overall system performance; ii) dynamic interactions with other active components of the MVDC grid (i.e, harmonic instability issues) are avoided [26].

Fig. 12 shows results focused on the high frequency model. Fig. 12(a) shows the resonant behavior around $\omega_{c}$, which is expected from the second order response in (19). Fig. 12(b) is included in order to support the assumption about ineffectiveness of the control action at high frequencies.

Fig. 13 represents the curves obtained for a reduced LVDC bus [i.e., $\bar{v}_{1}(t)<V_{1}$ ], which in wind energy is the typical scenario for low power generation [8], [37]. These results 


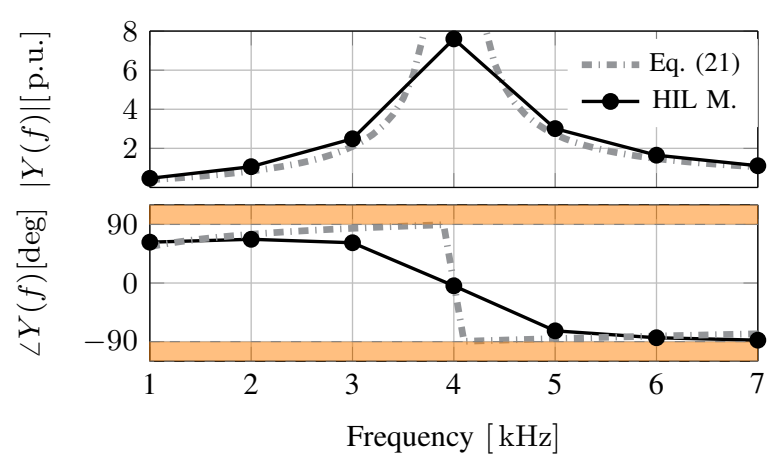

(a)

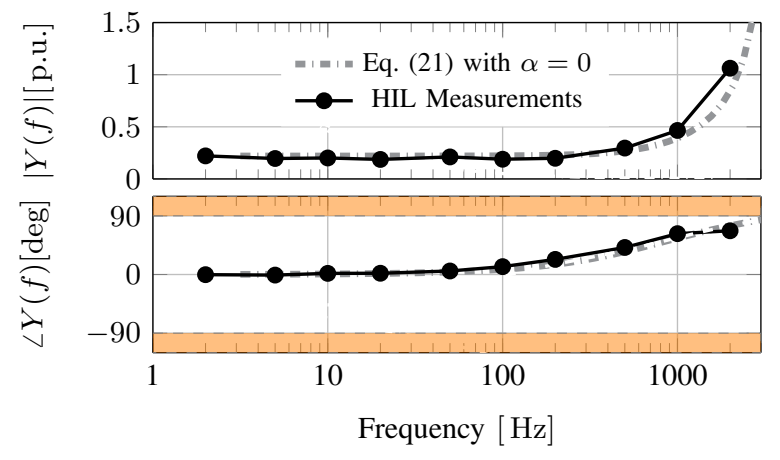

(b)

Fig. 12. Experimental results focused on the high frequency behavior (a) Admittance measurements around the Nyquist frequency, i.e., $4 \mathrm{kHz}$ (b) Admittance measurements of the open loop operation.

show that the power controller naturally adapts to the LVDC operating point and the passivity compliance is kept.

Figs. 14 show, in the time domain, the responses to an input perturbation at $v_{2}(t)$. It can be checked that, inside its bandwidth, the controller is able to attenuate low frequency oscillations. However, the controller has little effect on components of a frequency outside its theoretical bandwidth. Fig. 15 shows the effect when a $300 \mathrm{~Hz}$ ripple is programmed in $v_{1}(t)$ in order to mimic LVDC ripple due to passive rectification. As this frequency is outside the controller bandwidth, the effect is analogous to the one represented in Fig. 14(b).

\section{CONCLUSIONS}

This paper addresses the stability properties of a DAB based system aimed to interface LV distributed generation with MVDC grids. Detailed design guidelines for the controller are elaborated and provided. Analytical derivation of the admittance, as seen from the MVDC point of connection, is provided (accurate for all frequencies of the spectrum). Real time HIL verification using a detailed switched physical system confirms the accuracy of the theoretical modeling. Passivity compliance is employed as key figure of merit to assess the system from a system-level integration point of view. The results are really positive for the emerging MVDC technologies and systems: passivity compliance is achieved with a simple implementation (e.g., active damping or similar complex strategies have not been implemented) and a down-sampling strategy that restrains

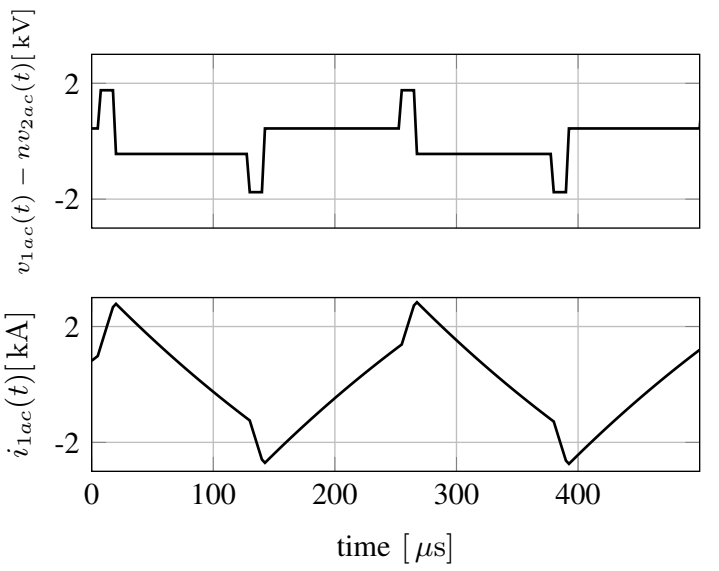

(a)

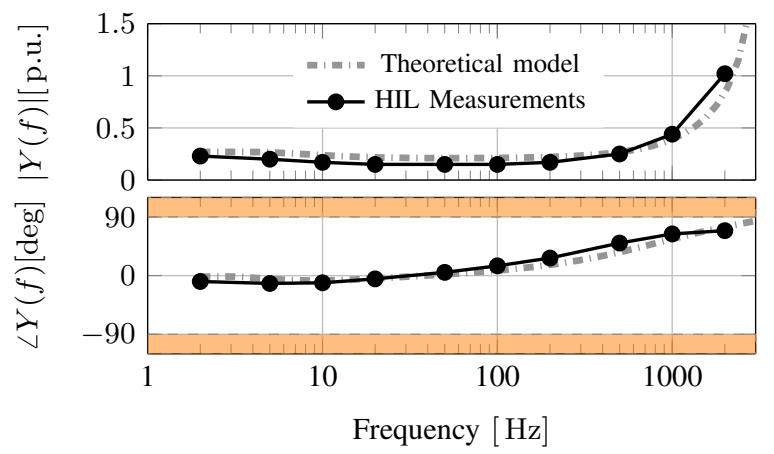

(b)

Fig. 13. Experimental results at light load and reduced LV bus. (a) Steady-state waveforms working at rated power $\left(P_{\text {ref }}=0.5 \mathrm{MW}, \bar{v}_{1}(t)=0.6 V_{1}, \Phi=16 \mathrm{deg}\right)$. (b) Measured admittance versus mathematical model from (21).

the effective control action in the low frequency range, taking advantage of the inherent passive properties of the DAB circuit at higher frequencies.

\section{APPENDIX}

This appendix provides an extended analysis of the conditions for passivity compliance of $Y_{2}(j \omega)$ as defined in (22). Firstly, by a change of variable $s=j \omega$ in (22),

$$
Y_{2}(j \omega)=\frac{-I_{2}}{V_{2}} \frac{j \omega\left[\frac{4-\pi}{4}\right]+2 \alpha e^{-j \omega T_{s 2}}}{j \omega+2 \alpha e^{-j \omega T_{s 2}}} .
$$

With some basic manipulations $Y_{2}(j \omega)$ can be expressed as

$$
Y_{2}(j \omega)=\operatorname{Real}\left\{Y_{2}(\omega)\right\}+j \operatorname{Imag}\left\{Y_{2}(\omega)\right\} .
$$

To do so, the numerator and denominator of (26) are multiplied by the complex conjugate of the denominator. Subsequently, the Euler formula is used to substitute $e^{ \pm j \omega T_{s 2}}$ terms by sine/cosine functions. The resulting expressions are

$$
\operatorname{Real}\left\{Y_{2}(\omega)\right\}=\frac{-I_{2}}{V_{2}} \frac{\frac{4-\pi}{4} \omega^{2}+4 \alpha^{2}-\left(\frac{8-\pi}{2}\right) \alpha \omega \sin \left(\omega T_{s 2}\right)}{\omega^{2}+4 \alpha^{2}-4 \alpha \omega \sin \left(\omega T_{s 2}\right)}
$$

and

$$
\operatorname{Imag}\left\{Y_{2}(\omega)\right\}=\frac{-I_{2}}{V_{2}} \frac{\left(\frac{-\pi}{2}\right) \alpha \omega \cos \left(\omega T_{s 2}\right)}{\omega^{2}+4 \alpha^{2}-4 \alpha \omega \sin \left(\omega T_{s 2}\right)}
$$




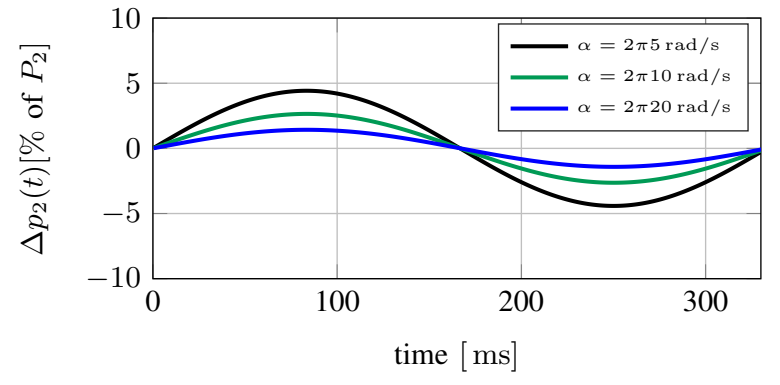

(a)

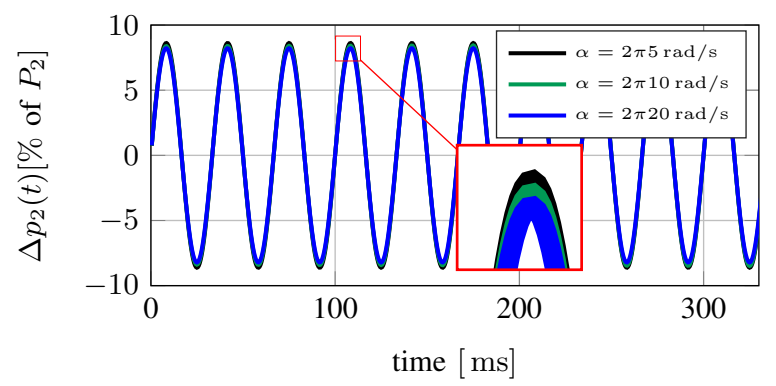

(b)

Fig. 14. Power response to a $10 \%$ amplitude perturbation in $v_{2}(t)$, obtained for different controller bandwidths (a clean $\Delta p_{2}\left(t, \omega_{p}\right)$ measurement is obtained by the FFT selective filtering method of Fig. 10). (a) Response to an input perturbation oscillating at $3 \mathrm{~Hz}$. (b) Response to an input perturbation oscillating at $30 \mathrm{~Hz}$.

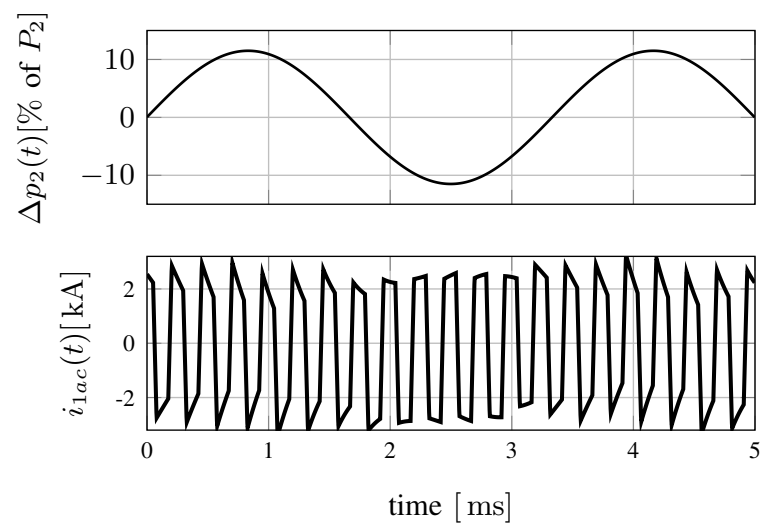

Fig. 15. Effect of a $10 \%$ amplitude, $300 \mathrm{~Hz}$ ripple in $v_{1}(t)$ (rated power).

From basic property of complex numbers, the denominator of (28) [and (29)] is positive. Furthermore, according to the current direction employed [see Fig. 3(a)], $I_{2}<0$. Therefore, the passivity condition is achieved if

$$
\frac{4-\pi}{4} \omega^{2}+4 \alpha^{2}-\left(\frac{8-\pi}{2}\right) \alpha \omega \sin \left(\omega T_{s 2}\right)>0 \forall \omega
$$

A solution to this inequality is complex to find analytically, but easy to solve by numerical methods (e.g., by a Matlab script). It has been found that equation (24) provides an optimistic estimation and should be corrected by a 0.852 gain. Regardless, once numerical/graphical methods are considered, evaluation of the complete admittance model in (21) is more accurate (e.g., analysis based on inspection of Fig. 6).

\section{RefERENCES}

[1] M. Stieneker, N. R. Averous, N. Soltau, H. Stagge, and R. W. D. Doncker, "Analysis of wind turbines connected to medium-voltage DC grids," in Proc. 16th European Conf. Power Electronics and Applications, pp. 1-10, Aug. 2014.

[2] J. E. Huber and J. W. Kolar, "Applicability of solid-state transformers in todays and future distribution grids," IEEE Trans. Smart Grid, vol. PP, no. 99 , p. 1, 2017.

[3] U. Javaid, F. D. Freijedo, D. Dujic, and W. van der Merwe, "Dynamic assessment of source-load interactions in marine MVDC distribution," IEEE Trans. Ind. Electron., vol. 64, no. 6, pp. 4372-4381, Jun. 2017.

[4] E. Rodriguez-Diaz, F. Chen, J. C. Vasquez, J. M. Guerrero, R. Burgos, and D. Boroyevich, "Voltage-level selection of future two-level lvdc distribution grids: A compromise between grid compatibility, safety, and efficiency," IEEE Electrif. Mag., vol. 4, no. 2, pp. 20-28, Jun. 2016.

[5] M. Liserre, R. Cardenas, M. Molinas, and J. Rodriguez, "Overview of multi-mw wind turbines and wind parks," IEEE Trans. Ind. Electron. vol. 58, no. 4, pp. 1081-1095, Apr. 2011.

[6] L. Sainz, L. Monjo, J. Pedra, M. Cheah-Mane, J. Liang, and O. GomisBellmunt, "Effect of wind turbine converter control on wind power plant harmonic response and resonances," IET Electric Power Applications, vol. 11, no. 2, pp. 157-168, 2017.

[7] C. Dincan, P. Kjaer, Y. hsing Chen, S.-M. Nielsen, and C. L. Bak, "Analysis and design of a series resonant converter with wide operating range and minimized transformer ratings," in Proceedings of 19th European Conference on Power Electronics and Applications (EPE'17 ECCE EUROPE), 2017.

[8] C. Dincan, P. Kjaer, Y. hsing Chen, S.-M. Nielsen, and C. L. Bak, "Selection of $\mathrm{dc} / \mathrm{dc}$ converter for offshore wind farms with mvdc power collection," in Proceedings of 19th European Conference on Power Electronics and Applications (EPE'17 ECCE EUROPE), 2017.

[9] H. Polinder, F. F. A. van der Pijl, G. J. de Vilder, and P. J. Tavner, "Comparison of direct-drive and geared generator concepts for wind turbines," IEEE Trans. Energy Convers., vol. 21, no. 3, pp. 725-733, Sep. 2006.

[10] N. Soltau, H. Stagge, R. W. D. Doncker, and O. Apeldoorn, "Development and demonstration of a medium-voltage high-power DC-dc converter for DC distribution systems," in Proc. IEEE 5th Int. Symp. Power Electronics for Distributed Generation Systems (PEDG), pp. 18, Jun. 2014.

[11] M. Stieneker, J. Riedel, N. Soltau, H. Stagge, and R. W. D. Doncker, "Design of series-connected dual-active bridges for integration of wind park cluster into mvdc grids," in Proc. 16th European Conf. Power Electronics and Applications, pp. 1-10, Aug. 2014.

[12] S. Cui, N. Soltau, and R. W. D. Doncker, "A high step-up ratio softswitching DC-dc converter for interconnection of mvdc and HVDC grids," IEEE Trans. Power Electron., vol. PP, no. 99, p. 1, 2017.

[13] J. E. Huber and J. W. Kolar, "Solid-state transformers: On the origins and evolution of key concepts," IEEE Industrial Electronics Magazine, vol. 10, no. 3, pp. 19-28, Sep. 2016.

[14] M. Mogorovic and D. Dujic, "Medium frequency transformer design and optimization," in Proc. Renewable Energy and Energy Management PCIM Europe 2017; Int. Exhibition and Conf. for Power Electronics, Intelligent Motion, pp. 1-8, May. 2017.

[15] D. Dujic, C. Zhao, A. Mester, J. K. Steinke, M. Weiss, S. LewdeniSchmid, T. Chaudhuri, and P. Stefanutti, "Power electronic traction transformer-low voltage prototype," IEEE Trans. Power Electron., vol. 28 , no. 12 , pp. 5522-5534, Dec. 2013.

[16] I. Villar, L. Mir, I. Etxeberria-Otadui, J. Colmenero, X. Agirre, and T. Nieva, "Optimal design and experimental validation of a mediumfrequency $400 \mathrm{kVA}$ power transformer for railway traction applications," in Proc. IEEE Energy Conversion Congress and Exposition (ECCE), pp. 684-690, Sep. 2012.

[17] A. K. Tripathi, K. Mainali, D. C. Patel, A. Kadavelugu, S. Hazra, S. Bhattacharya, and K. Hatua, "Design considerations of a $15-\mathrm{kV}$ SiC IGBT-based medium-voltage high-frequency isolated DC-DC converter," IEEE Trans. Ind. Appl., vol. 51, no. 4, pp. 3284-3294, 2015.

[18] A. Tripathi, S. Madhusoodhanan, K. M. K. Vechalapu, R. Chattopadhyay, and S. Bhattacharya, "Enabling DC microgrids with direct MV DC interfacing DAB converter based on $15 \mathrm{kV}$ SiC IGBT and $15 \mathrm{kV}$ SiC MOSFET," in Proc. of IEEE ECCE, 2016.

[19] M. Leibl, G. Ortiz, and J. W. Kolar, "Design and experimental analysis of a medium-frequency transformer for solid-state transformer applications," IEEE Journal of Emerging and Selected Topics in Power Electronics, vol. 5, no. 1, pp. 110-123, Mar. 2017. 
[20] J. Robinson, D. Jovcic, and G. Joos "Analysis and design of an offshore wind farm using a MV DC grid," IEEE Trans. Power Deliv., vol. 25, no. 4, pp. 2164-2173, 2010.

[21] S. Vogel, "Investigation of DC Collection Networks for Offshore Wind Farms," Technical University of Denmark, Tech. Rep., 2014.

[22] U. Javaid, D. Dujic, and W. van der Merwe, "MVDC marine electrical distribution: Are we ready?" in Proc. of IEEE IECON, Yokohama, Japan, Nov. 2015.

[23] C. Yuan, M. A. Haj-ahmed, and M. S. Illindala, "Protection strategies for medium-voltage direct-current microgrid at a remote area mine site," IEEE Trans. Ind. Appl., vol. 51, no. 4, pp. 2846-2853, Jul. 2015.

[24] A. Yanushkevich and J. Švec, "Multi-feeder protection system with hybrid circuit breakers for mvdc grids," Electric Power Components and Systems, vol. 45, no. 10, pp. 1130-1140, 2017

[25] C. Peng, I. Husain, A. Q. Huang, B. Lequesne, and R. Briggs, "A fast mechanical switch for medium-voltage hybrid dc and ac circuit breakers," IEEE Trans. Ind. Appl., vol. 52, no. 4, pp. 2911-2918, 2016.

[26] E. Mollerstedt and B. Bernhardsson, "Out of control because of harmonics-an analysis of the harmonic response of an inverter locomotive," IEEE Control Syst. Mag., vol. 20, no. 4, pp. 70-81, 2000

[27] M. Jansson, A. Danielsson, J. Galic, K. Pietiläinen, and L. Harnefors, "Stable and passive traction drives," in Proc. IEEE Nordic Power Ind. Electron. Conf., pp. 1-6, 2004

[28] L. Harnefors, M. Bongiorno, and S. Lundberg, "Input-admittance calculation and shaping for controlled voltage-source converters," IEEE Trans. Ind. Electron., vol. 54, no. 6, pp. 3323-3334, 2007.

[29] J. L. Agorreta, M. Borrega, J. Lopez, and L. Marroyo, "Modeling and control of n-paralleled grid-connected inverters with lcl filter coupled due to grid impedance in PV plants," IEEE Trans. Power Electron. vol. 26, no. 3, pp. 770-785, Mar. 2011.

[30] F. Wang, J. L. Duarte, M. A. M. Hendrix, and P. F. Ribeiro, "Modeling and analysis of grid harmonic distortion impact of aggregated $\mathrm{dg}$ inverters," IEEE Trans. Power Electron., vol. 26, no. 3, pp. 786-797, Mar. 2011.

[31] A. Riccobono and E. Santi, "Comprehensive review of stability criteria for DC power distribution systems," IEEE Trans. Ind. Appl., vol. 50, no. 5, pp. 3525-3535, Sep. 2014.

[32] L. Harnefors, A. G. Yepes, A. Vidal, and J. Doval-Gandoy, "Passivitybased controller design of grid-connected vscs for prevention of electrical resonance instability," IEEE Trans. Ind. Electron., vol. 62 , no. 2 , pp. 702-710, 2015 .

[33] F. D. Freijedo, D. Dujic, and J. A. Marrero-Sosa, "Design for passivity in the z-domain for LCL grid-connected converters," in Proc. of the IEEE Industrial Electronics Society Annual Conference, pp. 7016-7021, Firenze, Italy, Oct. 2016

[34] L. Harnefors, R. Finger, X. Wang, H. Bai, and F. Blaabjerg, "Vsc input-admittance modeling and analysis above the Nyquist frequency for passivity-based stability assessment," IEEE Trans. Ind. Electron., vol. 64, no. 8, pp. 6362-6370, Aug. 2017

[35] J. Sun, "Autonomous local control and stability analysis of multiterminal dc systems," IEEE Trans. Emerg. Sel. Topics Power Electron., vol. 3, no. 4, pp. 1078-1089, Dec. 2015

[36] Q. Ye, R. Mo, and H. Li, "Low-frequency resonance suppression of a Dual Active Bridge (DAB) DC/DC converter Enabled DC Microgrid with Constant Power Loads (CPLs) Based on Reduced-Order Impedance Models," IEEE Trans. Emerg. Sel. Topics Power Electron., 2017.

[37] E. Van der Hooft, P. Schaak, and T. Van Engelen, "Wind turbine control algorithms," TU Delft., Tech. Rep., 2003.

[38] F. Krismer and J. W. Kolar, "Accurate small-signal model for the digital control of an automotive bidirectional dual active bridge," IEEE Trans. Power Electron., vol. 24, no. 12, pp. 2756-2768, 2009.

[39] F. Krismer, "Modeling and optimization of bidirectional dual active bridge dc-dc converter topologies," PhD dissertation, ETH Zurich, 2011.

[40] F. D. Freijedo, A. Vidal, A. G. Yepes, J. M. Guerrero, O. Lopez, J. Malvar, and J. Doval-Gandoy, "Tuning of synchronous-frame PI current controllers in grid-connected converters operating at a low sampling rate by MIMO root locus," IEEE Trans. Ind. Electron. vol. 62 , no. 8, pp. 5006-5017, 2015

[41] R. N. Bracewell, The Fourier transform and its Applications (Third Edition). McGraw-Hill, 2000

[42] H. Mouton and B. Putzeys, "Understanding the PWM nonlinearity: Single-sided modulation," IEEE Trans. Power Electron., vol. 27, no. 4, pp. 2116-2128, Apr. 2012

[43] H. d. T. Mouton, B. McGrath, D. G. Holmes, and R. H. Wilkinson, "One-dimensional spectral analysis of complex PWM waveforms using superposition," IEEE Trans. Power Electron., vol. 29, no. 12, pp. 67626778, Dec. 2014
[44] M. H. Hansen, A. Hansen, T. J. Larsen, S. Oye, P. Sorensen, and P. Fuglsang, "Control design for a pitch-regulated, variable speed wind turbine. Riso-R-1500(EN)," Riso National Laboratory, Tech. Rep., 2005.

[45] F. D. Freijedo, J. Doval-Gandoy, O. Lopez, and C. Martinez-Penalver, "New algorithm for grid synchronization based on fourier series," in Proc. of EPE Conference, Aalborg, Denmark, Sep. 2007.

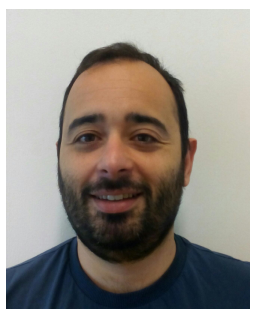

Francisco D. Freijedo (M'07-SM'16) received the M.Sc. degree in physics from the University of Santiago de Compostela, Santiago de Compostela, Spain, in 2002 and the Ph.D. degree in Electrical Engineering from the University of Vigo, Vigo, Spain, in 2009. From 2005 to 2011 he was a Lecturer in the Department of Electronics Technology, University of Vigo. From 2011 to 2014, he worked in Gamesa Innovation and Technology as a Power Electronics Control Engineer, where he was involved in Wind Energy projects. From 2014 to 2016, he was a Postdoctoral Researcher in the Department of Energy Technology, Aalborg University. Since 2016, he is a Scientific Collaborator of the Power Electronics Laboratory, Ecole Polytechnique Federale de Lausanne. His research interests include many power conversion technologies and challenging control problems.

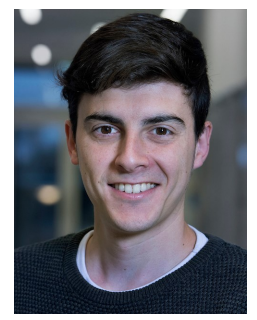

Enrique Rodriguez (S'15-M'18) received the B.Sc. and Msc degrees in Electronics Engineering at the University of Oviedo, Oviedo, Spain, in 2012 and 2014,respectively. He obtained his PhD degree in Power Electronics from Aalborg Universitet, Denmark, in 2018, where currently is a Postdoctoral Researcher. In 2017, he was a guest researcher at the Power Electronic Laboratory at EPFL. He is a member of the International Electrotechnical Commission System Evaluation Group SEG4 on Low Voltage DC Applications, Distribution, and Safety for Use in Developed and Developing Economies. His research interests include DC distribution systems, control of power converters and microgrids.

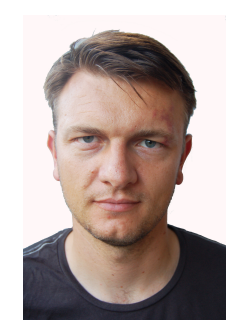

Drazen Dujic (S'03-M'09-SM'12) received the Dipl.-Ing. and M.Sc. degrees from the University of Novi Sad, Novi Sad, Serbia, in 2002 and 2005 respectively, and the Ph.D. degree from Liverpool John Moores University, Liverpool, U.K., in 2008, all in Electrical Engineering. From 2002 to 2006, he was a Research Assistant with the Department of Electrical Engineering, University of Novi Sad. From 2006 to 2009, he was a Research Associate with Liverpool John Moores University. From 2009 to 2013, he was with the ABB Corporate Research Center, Switzerland, as a Principal Scientist working on Power Electronics Projects. During 2010-2011, he was involved in the development of the Power Electronic Traction Transformer (PETT). From 2013 to 2014, he was with ABB Medium Voltage Drives, Turgi, Switzerland, as an R\&D Platform Manager. He is currently an Assistant Professor with the Ecole Polytechnique Federale de Lausanne, Lausanne, Switzerland, where he is also the Director of the Power Electronics Laboratory. He has authored/coauthored more than 100 scientific publications and has filed 11 patents. His current research interests include the areas of design and control of advanced highpower electronics systems and high-performance drives. Dr. Dujic is an Associate Editor for the IEEE TRANSACTIONS ON INDUSTRIAL ELECTRONICS, IEEE TRANSACTIONS ON POWER ELECTRONICS, and IET Electric Power Applications. He received the First Prize Paper Award form the Electrical Machines Committee of the IEEE IES at the IECON 2007. In 2014, he received the Isao Takahashi Power Electronics Award for outstanding achievement in power electronics. 